\title{
Completing the Basic and Clinical Science Course as a First-Year Ophthalmology Resident
}

\author{
Isdin Oke, MD ${ }^{1}$ Nicole H. Siegel, MD ${ }^{1}$ \\ Jean E. Ramsey, MD, MPH ${ }^{1}$ \\ ${ }^{1}$ Department of Ophthalmology, Boston Medical Center, Boston \\ University, Boston, Massachusetts \\ J Acad Ophthalmol 2019;11:e54-e58.
}

Crandall E. Peeler, MD ${ }^{1}$

Steven

Address for correspondence Isdin Oke, MD, Department of Ophthalmology, Boston Medical Center, 850 Harrison Ave 3rd Floor, Boston, MA 02118 (e-mail: Isdin.Oke@bmc.org).

\begin{abstract}
Keywords

- Basic Clinical Science Course

- Ophthalmology Knowledge

Assessment Program

- reading speed

- study schedule
\end{abstract}

Background The Basic and Clinical Science Course (BCSC) is the primary educational curriculum for ophthalmology resident physicians in the United States. The Ophthalmic Knowledge Assessment Program (OKAP) examination is an annual evaluation completed by residents that is based primarily on the BCSC curriculum. First-year ophthalmology residents are encouraged to complete the 13 volume BCSC series in preparation for the OKAP examination while balancing a steep clinical learning curve and substantial call schedule. By calculating the daily time commitment necessary to read each volume in the series, we hope to help residents create a realistic study plan to compete the entire BSCS series before the OKAP examination.

Methods We determine the word counts of each volume using an electronic copy of the 2018-2019 BCSC series. We include all text sections and legends, and we exclude all figures and tables. We calculate the time per day of dedicated reading required to complete a goal number of BCSC books between the start of ophthalmology residency (postgraduate year 2 [PGY2]) and the OKAP examination by developing a formula that is a function of self-assessed reading speed.

Results A first-year ophthalmology (PGY2) resident with an average reading speed of 250 words per minute must read for 25.0 minutes per day to complete the entire BCSC series before the OKAP examination. If studying is initiated at the beginning of intern (PGY1) year, the resident must read for 10.2 minutes per day. We introduce a formula and provide a table to guide residents on the amount of time needed to dedicate to reading the BCSC each day as a function of self-assessed reading speed.

Discussion Completion of all volumes of the BCSC requires a daily commitment with little room for missed sessions. The commitment is substantially more realistic if initiated during the PGY1 year; thus, residency programs should encourage an early start to OKAP preparation. We hope with a better understanding of the daily time commitment involved in completing the BCSC series, ophthalmology residents will be able to develop more successful study plans.
The Basic and Clinical Sciences Course (BCSC) is an educational resource updated annually by the American Academy of Ophthalmology (AAO) that serves as the primary reference tool for ophthalmology physicians in training in the United
States. The BSCS has been available for over 70 years, and the current edition consists of 13 volumes, each focusing on a specific subspecialty of ophthalmology. ${ }^{1}$ Importantly, the $\mathrm{BCSC}$ serves as a reference manual for the annual Ophthalmic received

September 20, 2019 accepted after revision November 4, 2019
DOI https://doi.org/

$10.1055 / \mathrm{s}-0039-3401848$. ISSN 2475-4757.
Copyright $\odot 2019$ by Thieme Medical

Publishers, Inc., 333 Seventh Avenue, New York, NY 10001, USA. Tel: +1(212) 584-4662.
License terms

() (1) $\Theta \circledast$ 
Knowledge Assessment Program (OKAP) and the Written Qualifying Exam (WQE) of the American Board of Ophthalmology (ABO). ${ }^{2}$

The OKAP is a computer-based multiple-choice examination that is administered to all ophthalmology residents in the United States and Canada. ${ }^{3-5}$ It is designed to measure the ophthalmic knowledge of residents relative to their peers and is completed annually to facilitate the ongoing assessment of resident progress and program effectiveness. Performance on the OKAP examination is often used as evaluation criteria on fellowship applications ${ }^{6}$ and has been correlated with ability to successfully pass the written and oral ophthalmic boards. ${ }^{2,3,7}$

Questions for the OKAP examination are derived from the content of the $\mathrm{BCSC}^{8}$ and traditionally, ophthalmology residency programs emphasize the use of the BCSC series in preparation for the exams. However, it may be challenging for residents to complete the entire series before the OKAP examination given the large corpus of ophthalmic knowledge that is increasing each year. ${ }^{1}$ Furthermore, there are many other resources such as review manuals, residency program lectures, and question banks that provide alternative methods for preparation for the examination and compete for the available study time. ${ }^{9}$

There is a finite amount of preparation time-residents start their ophthalmology training in the second year of the postgraduate medical education (postgraduate year 2 [PGY2]) and have just over 8 months between the start date (July) and the traditional timing of the OKAP examination (mid-March). Ophthalmology residents are encouraged to start their preparation early, and there are several online resources with study outlines suggesting the number of sections/pages to read each week. ${ }^{10}$ Unfortunately, there is limited guidance on how much time needs to be dedicated to completing the BCSC series. Access to such information would be valuable in balancing the challenge of studying for the examination while simultaneously learning new clinical skills and juggling a busy call schedule.

In this investigation, we hope to answer the following question: what is the minimum daily time commitment for a first-year ophthalmology resident to complete the BCSC series before the OKAP examination? We hope to develop a model that can provide both incoming and current ophthalmology residents with an additional tool to manage their time and guide their ophthalmology education.

\section{Methods}

In this study, we develop a model for the daily time commitment required to read the BSCS series. We use an electronic copy of the 2018-2019 BCSC to calculate the cumulative word count in all text sections for each volume. We include all text sections including titles, paragraphs, figure legends, footnotes, and self-assessment questions. We exclude any images with text, as well as any diagrams with text, such as flow charts and tables. We include only the educational content by excluding all text before the first chapter and all text after the end of the last chapter in each volume.

Raw text data was imported and processed using MATLAB Version R2018B (MathWorks, Natick, MA). The mean and range in word counts of each section were calculated using $R$ Studio for Mac Version 1.2.1335 (R Studio, Boston, MA).

We generate a formula to calculate minimal daily reading requirements based on cumulative text length of each BCSC volume and self-assessed reading speed. The primary outcome in this investigation is the minimum required reading time per day as a function of reading speed for a first-year ophthalmology resident to complete the BCSC series before the annual OKAP examination. We define the start date as July 1 st and the end date as March 23rd based on dates from the 2018 to 2019 academic year. Secondary outcomes include daily time commitment to complete the BCSC given alternative start dates, such as the beginning of the PGY1 year.

\section{Results}

The length of all text sections in the entire BCSC series is $\sim 1.35$ million words. The longest volume is "External Disease and Cornea," and the shortest volume is "Lens and Cataract" with $\sim 130,000$ and 70,000 total words, respectively. Assuming a constant reading speed of 250 words per minute, it would take the average reader 89.2 hours to complete all of the text sections of the BCSC series ( - Table 1). For a first-year resident studying 6 days per week, this equates to 25.0 minutes of reading per day starting July 1 st and ending a week before the OKAP examination. The minimum daily reading requirement can be calculated by:

$$
T=C v(1)
$$

$T$ is the time per day reading in minutes, $v$ is the reading speed in words per minute, and $C$ is a constant based on the

Table 1 Time to complete each book in the BCSC series assuming a constant reading speed of 250 words per minute

\begin{tabular}{|l|l|l|}
\hline No. & Topic & $\begin{array}{l}\text { Estimated time } \\
\text { to read (hours) }\end{array}$ \\
\hline $\mathbf{1}$ & General Medicine & 7.9 \\
\hline $\mathbf{2}$ & $\begin{array}{l}\text { Fundamentals and Principles } \\
\text { of Ophthalmology }\end{array}$ & 8.1 \\
\hline $\mathbf{3}$ & Clinical Optics & 7.3 \\
\hline $\mathbf{4}$ & $\begin{array}{l}\text { Ophthalmic Pathology and } \\
\text { Intraocular Tumors }\end{array}$ & 5.4 \\
\hline $\mathbf{5}$ & Neuro-Ophthalmology & 7.9 \\
\hline $\mathbf{6}$ & $\begin{array}{l}\text { Pediatric Ophthalmology } \\
\text { and Strabismus }\end{array}$ & 8.1 \\
\hline $\mathbf{7}$ & $\begin{array}{l}\text { Orbit, Eyelids, and } \\
\text { Lacrimal System }\end{array}$ & 6.0 \\
\hline $\mathbf{8}$ & External Disease and Cornea & 8.9 \\
\hline $\mathbf{9}$ & $\begin{array}{l}\text { Intraocular Inflammation } \\
\text { and Uveitis }\end{array}$ & 6.9 \\
\hline 10 & Glaucoma & 4.9 \\
\hline 11 & Lens and Cataract & 4.8 \\
\hline 12 & Retina and Vitreous & 8.1 \\
\hline 13 & Refractive Surgery & 4.9 \\
\hline
\end{tabular}

Abbreviation: BCSC, Basic and Clinical Science Course. 
Table 2 The daily time commitment required for reading the BSCS series

\begin{tabular}{|l|l|l|l|l|l|l|l|}
\hline \multirow{2}{*}{ Goal number of books } & \multicolumn{6}{l}{ Reading speed (words/min) } \\
\cline { 2 - 9 } & 100 & $\mathbf{1 5 0}$ & $\mathbf{2 0 0}$ & $\mathbf{2 5 0}$ & $\mathbf{3 0 0}$ & $\mathbf{3 5 0}$ & $\mathbf{4 0 0}$ \\
\hline $\mathbf{1}$ & 4.8 & 3.2 & 2.4 & 1.9 & 1.6 & 1.3 & 1.2 \\
\hline $\mathbf{2}$ & 9.6 & 6.4 & 4.8 & 3.8 & 3.2 & 2.7 & 2.4 \\
\hline $\mathbf{3}$ & 14.4 & 9.6 & 7.2 & 5.7 & 4.8 & 4.1 & 3.6 \\
\hline $\mathbf{4}$ & 19.3 & 12.8 & 9.6 & 7.7 & 6.4 & 5.5 & 4.8 \\
\hline $\mathbf{5}$ & 24.1 & 16 & 12 & 9.6 & 8 & 6.8 & 6 \\
\hline $\mathbf{6}$ & 28.9 & 19.3 & 14.4 & 11.5 & 9.6 & 8.2 & 7.2 \\
\hline $\mathbf{7}$ & 33.7 & 22.5 & 16.8 & 13.5 & 11.2 & 9.6 & 8.4 \\
\hline $\mathbf{8}$ & 38.6 & 25.7 & 19.3 & 15.4 & 12.8 & 11 & 9.6 \\
\hline $\mathbf{9}$ & 43.4 & 28.9 & 21.7 & 17.3 & 14.4 & 12.4 & 10.8 \\
\hline $\mathbf{1 0}$ & 48.2 & 32.1 & 24.1 & 19.3 & 16 & 13.7 & 12 \\
\hline $\mathbf{1 1}$ & 53 & 35.3 & 26.5 & 21.2 & 17.6 & 15.1 & 13.2 \\
\hline $\mathbf{1 2}$ & 57.9 & 38.6 & 28.9 & 23.1 & 19.3 & 16.5 & 14.4 \\
\hline $\mathbf{1 3}$ & 62.7 & 41.8 & 31.3 & 25 & 20.9 & 17.9 & 15.6 \\
\hline
\end{tabular}

Abbreviations: BCSC, Basic and Clinical Science Course; OKAP, Ophthalmic Knowledge Assessment Program.

This table shows the time required (in minutes per day) for a first-year ophthalmology resident starting July 1 st to complete a goal number of books before the OKAP examination in March. With a self-assessed reading speed (in words per minute), this table can be used as a tool to design a study schedule.

BCSC text length. We can further generalize this formula to allow for individual specialization of study plan as follows:

$$
T=C \cdot v \cdot B \cdot D(\Delta t-h)(2)
$$

$B$ is the goal number of books to be covered, $D$ is the number of days per week dedicated to studying, $\delta$ t is the amount of time to study in days, and $h$ is number of anticipated days off for vacation and/or holidays. The constant $\mathrm{C}$ in the generalized formula equals 14,835. - Table 2 summarizes daily reading time as a function of the goal number of books covered and self-assessed reading speed. To maximize ease of use, both - Table 2 and Eq. 2 assume each volume in the series is of equal length ( 1.35 million words/13 volumes). - Table 3 demonstrates reading times based on alternative starting dates as determined by the generalized formula. The time for a resident with a reading speed of 250 words/minute to complete the BCSC series if started in the PGY 1 year is 10.2 minutes per day. -Figure 1 illustrates a sample reading timeline for completing the series prior to the OKAP examination based on the length of each volume.

\section{Discussion}

In this analysis, we show that the text sections of the entire BSCS can be completed at a sustained reading speed of 250 words per minute in $\sim 90$ hours. This time period translates to 25 minutes per day of dedicated BCSC reading for a firstyear resident to complete the entire series before the OKAP examination. At first glance, this commitment seems like a reasonably attainable goal; however, given the caveats discussed below, we emphasize that these numbers can at best be interpreted as the minimum amount of time necessary to complete all of the volumes.

First, we are using a text-centric approach to calculate the length of each BCSC volume and base our model on the cumulative word count. We chose this approach because it provides a more accurate prediction of required reading time compared with calculating the number of pages per section and pages per minute given the high variability in amount of content in each individual page. By excluding images, figures,

Table 3 Alternative strategies for reading the entire BCSC series

\begin{tabular}{|c|c|c|c|c|c|c|c|}
\hline \multirow[t]{2}{*}{ Goal } & \multicolumn{7}{|c|}{ Reading speed (words/min) } \\
\hline & 100 & 150 & 200 & 250 & 300 & 350 & 400 \\
\hline Entire BCSC series started PGY2 before OKAPs & 62.7 & 41.8 & 31.3 & 25 & 20.9 & 17.9 & 15.6 \\
\hline Entire BCSC series started PGY1 before OKAPs & 25.5 & 17 & 12.7 & 10.2 & 8.5 & 7.3 & 6.3 \\
\hline Entire BCSC series over course of 3-year residency & 14.3 & 9.5 & 7.1 & 5.7 & 4.7 & 4.1 & 3.5 \\
\hline Cramming entire BCSC series from Jan $1^{\text {st }}$ before OKAPs (no breaks) & 166.6 & 111.1 & 83.3 & 66.6 & 55.5 & 47.6 & 41.6 \\
\hline
\end{tabular}

Abbreviations: BCSC, Basic and Clinical Science Course; OKAPs, Ophthalmic Knowledge Assessment Programs; PGY1, postgraduate year 1. Note: Time spent (in minutes per day) reading to complete the BCSC series with the specified time frame goal. Notice that starting the BCSC series in the PGY1 intern year can make completing the series before the first OKAP examination substantially more feasible in terms of per day commitment. 


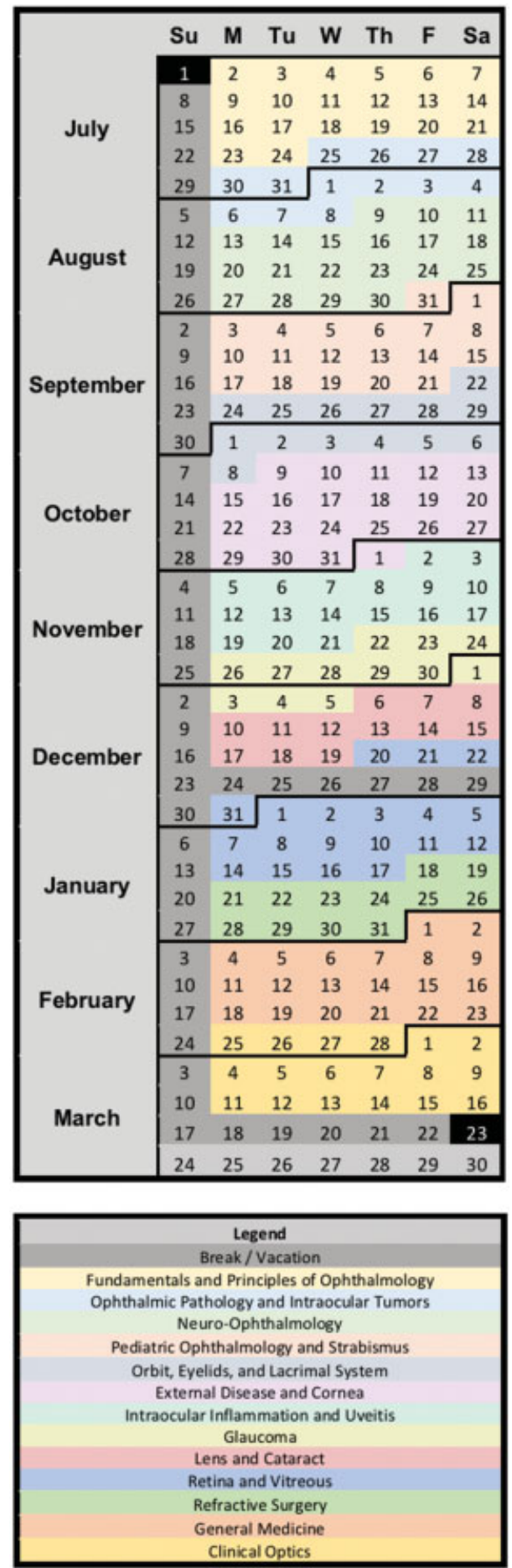

Fig. 1 Sample reading timeline for completing all Basic and Clinical Science Course books prior to the Ophthalmic Knowledge Assessment Program examination. Self-assessed reading speed can be used to calculate the amount of time needed to dedicate per day to follow such a schedule.

and tables from our calculation, however, we underestimate the time required to read each section. We intentionally exclude figures due to the challenge of assigning a "reading time" to a figure given both the high degree of inter-figure and inter-reader variability. Each figure is inherently different and requires varying levels of interpretation. In addition, each individual reader is unique and may prioritize time spent on figures differently. Further investigation may seek to quantify the inter-figure and inter-reader variability and assign a time-value as a function of figure complexity.

Next, our methodology relies on a sustained self-determined reading speed. Reading speed of the general population ranges between 200 and 300 words per minutes and increases as a function of level of education. ${ }^{11}$ One may also assume that reading speed is a function of the depth and complexity of the material. The combination of advanced text and terms and definitions that are likely novel to first-year ophthalmology residents would be expected to dramatically decrease the average reading speed. Furthermore, while reading the BCSC text, various retention techniques such as reading aloud, annotating the text, or taking notes by hand or on a computer will impact the net words covered per minute. To our knowledge, there have been no studies looking at the reading speed of ophthalmic texts by trainees of differing levels. For this reason, we propose that residents wishing to apply our model assess their own reading speed and use the table to predict how much time to dedicate to reading the BSCS each day. There are many tools available for self-evaluation of reading speed that residents could employ that mimic the reading level of the BCSC series. Using longer text and paragraphs would be preferable because of lower variance in measurements. ${ }^{12,13}$

Furthermore, we describe a single read through the BCSC series. In practice, certain sections may need to be read only once, whereas other sections may need to be reread multiple times to attain an adequate level of comprehension. To our knowledge, there have not been any published studies investigating the retention and recall in preparation for the OKAP examination. Our results should thus be interpreted as a guideline for the minimal time commitment required when using the BCSC as a preparation resource.

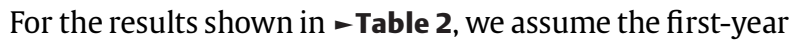
resident is studying 6 out of 7 days of the week with 1 week for a break/holiday with the aim of completing a goal number of books by 1 week before the examination. The method of preparation can vary for individuals, and for this reason, we provide an equation (Eq. 2) that can be used by individuals to tailor their own study plan. It is up to each individual to account for missed days, and it may be wise to incorporate additional days into the holiday variable to provide a buffer for unforeseen circumstances. We intentionally chose to simplify Eq. 2 and - Table 2 by assuming each textbook is the same average length. A more complex formula can be designed that takes into consideration the different lengths of each textbook; however, we felt the benefits of a simplicity and ease of use outweighed potential benefits of additional precision.

For a resident that starts preparing for the OKAP examination using the BCSC in the first-year of postgraduate medical training (PGY1), we show a required commitment of $\sim 10$ minutes per day or less than 1 hour per week (assuming a 6 days per week study schedule). We believe this is a much more realistic pace where even a few weeks of missed study time could be made up. We feel our study supports the growing sentiment to incorporate more ophthalmic training into the PGY1 year, prior to the official start of an ophthalmology residency in the PGY2 year. Residency programs should 
consider providing the BCSC to incoming residents as early as the PGY1 year and encourage them to begin reviewing the material immediately if it remains a priority to complete the curriculum by the OKAP examination.

In this investigation, we propose a tool to help guide both current and future residents in planning their coverage of the BSCS reading material. We hope that with a better understanding of the daily time commitment involved in completing the BCSC series both ophthalmology residents and residency programs will be able to develop more successful study plans and ultimately improved educational outcomes.

\section{Conflict of Interest}

None declared.

\section{References}

1 Brown SM, Freedman KA. The weight of the basic and clinical science course. Ophthalmology 2003;110(08):1672-1673

2 Johnson GA, Bloom JN, Szczotka-Flynn L, Zauner D, Tomsak RL. A comparative study of resident performance on standardized training examinations and the American Board of Ophthalmology Written Examination. Ophthalmology 2010;117(12):2435-2439

3 Lee AG, Oetting TA, Blomquist PH, et al. A multicenter analysis of the ophthalmic knowledge assessment program and American Board of Ophthalmology written qualifying examination performance. Ophthalmology 2012;119(10):1949-1953

4 Zafar S, Wang X, Srikumaran D, et al. Resident and program characteristics that impact performance on the Ophthalmic
Knowledge Assessment Program (OKAP). BMC Med Educ 2019; 19(01):190

5 Liesegang TJ. New directions for the Ophthalmic Knowledge Assessment Program (OKAP) examination. Ophthalmology 1994;101 (01):194-198

6 Kempton JE, Shields MB, Afshari NA, Dou W, Adelman RA. Fellow selection criteria. Ophthalmology 2009;116(05):10201020.e4

7 Chen PP. Resident OKAP performance. Ophthalmology 2012;119 (03):656

8 Rapuano, Christopher, Stout, Timothy, Ophthalmic Knowledge Assessment Program Committee. OKAP User Christop https:// www.aao.org/okap-exam. Accessed September 1st 2019

9 van Landingham SW, Srikumaran D, Siddiqui AA, Swenor B, Ramulu PY. Characteristics that predict ophthalmic knowledge among ophthalmology residents. Ophthalmology 2015;122(05): 1063-1065

10 Chan-Kai. Brian. OKAPs Reading Schedule. https://www.aao.org/ young-ophthalmologists/yo-info/article/first-year-resident-reading-guide. Accessed July 15, 2019

11 Rayner K, Schotter ER, Masson MEJ, Potter MC, Treiman R. So much to read, so little time.:31. Psychol Sci Public Interest 2016; 17(01):4-34

12 Altpeter EK, Marx T, Nguyen NX, Naumann A, Trauzettel-Klosinski S. Measurement of reading speed with standardized texts: a comparison of single sentences and paragraphs. Graefes Arch Clin Exp Ophthalmol 2015;253(08):1369-1375

13 Trauzettel-Klosinski S, Dietz K; IReST Study Group. Standardized assessment of reading performance: the New International Reading Speed Texts IReST. Invest Ophthalmol Vis Sci 2012;53(09): 5452-5461 